\title{
Philosophiques
}

\section{La politique du citoyen (sur un passage orphelin du Contrat social)}

\section{Bruno Bernardi}

Volume 43, numéro 1, printemps 2016

URI : https://id.erudit.org/iderudit/1036465ar

DOI : https://doi.org/10.7202/1036465ar

Aller au sommaire du numéro

\section{Éditeur(s)}

Société de philosophie du Québec

\section{ISSN}

0316-2923 (imprimé)

1492-1391 (numérique)

Découvrir la revue

Citer cet article

Bernardi, B. (2016). La politique du citoyen (sur un passage orphelin du Contrat social). Philosophiques, 43(1), 3-21. https://doi.org/10.7202/1036465ar
Résumé de l'article

L'analyse d'un passage peu commenté du Contrat social (III, IX) montre que Rousseau aborde d'une façon singulière la problématique aussi classique qu’actuelle des rapports entre liberté et sécurité. La position de sujet (membre de l'État) et celle de citoyen (membre du peuple souverain) découlent toutes deux de la nature du corps politique. Elles sont porteuses de deux types d'exigences : la protection des personnes et des biens dans un cas, celle de la liberté civile et politique dans l'autre. Rousseau n'oppose pas ces exigences entre elles mais distingue les deux ordres de priorité qu'elles rendent possibles. La politique du citoyen consiste à faire de la liberté la condition de la sécurité. 


\title{
La politique du citoyen (sur un passage orphelin du Contrat social)
}

\author{
BRUNO BERNARDI \\ bruno-bernardi@wanadoo.fr
}

\begin{abstract}
RÉSUMÉ. - L'analyse d'un passage peu commenté du Contrat social (III, IX) montre que Rousseau aborde d'une façon singulière la problématique aussi classique qu'actuelle des rapports entre liberté et sécurité. La position de sujet (membre de l'État) et celle de citoyen (membre du peuple souverain) découlent toutes deux de la nature du corps politique. Elles sont porteuses de deux types d'exigences: la protection des personnes et des biens dans un cas, celle de la liberté civile et politique dans l'autre. Rousseau n'oppose pas ces exigences entre elles mais distingue les deux ordres de priorité qu'elles rendent possibles. La politique du citoyen consiste à faire de la liberté la condition de la sécurité.
\end{abstract}

\begin{abstract}
The study of a little-commented passage of Rousseau's Social Contract shows that Rousseau tackles the connection between freedom and security from a rather original angle. Both the statuses of being a subject (member of the state) and being a citizen (member of the sovereign people) derive from the nature of the body politic and imply two kinds of requirements: the protection of persons and goods and that of civil and political liberty. Rousseau does not consider these requirements as conflicting but sees them as ordered in the following way: liberty should be the condition of security.
\end{abstract}

Rousseau parlait du Contrat social comme de son «traité de droit politique» et, par son sous-titre, indique clairement qu'il s'y place du point de vue des "principes ${ }^{1}$. Ce faisant, il nous autorise à y voir un ouvrage traitant des fondements de la politique. Mais nous devons aussi tenir compte du motif qu'il avance pour justifier sa rédaction. Une de ses thèses constantes est que "l'instruction qui n'a pas un but déterminé n'est rien ", et qu'on ne comprend que ce dont on a "un intérêt sensible à s'instruire "². L'Émile l'applique explicitement au droit politique: «La plus grande difficulté pour éclaircir ces importantes matières, c'est d'intéresser un particulier à les discuter, de répondre à ces deux questions: que m'importe? et, qu'y puis-je faire? $»^{3}$. Cette thèse innerve aussi le préambule du Contrat social où elle

1. Lorsque Rousseau annonce à son éditeur que son ouvrage est "enfin prêt", il en parle comme de son «traité de droit politique» (à M.-M. Rey, 9 août I76I, Leigh, Correspondance Complète, t. IX, $\mathrm{n}^{\circ}$ I47I). Dans l'édition de I762 titre et sous-titre ( Principes du droit politique») occupent une place égale, chacun une pleine page.

2. Émile, L. V, OC IV, p. 832. Sauf mention contraire, les références à Rousseau renverront à l'édition de ses CEuvres Complètes, collection de la Pléiade ( 5 volumes notés OC I à OC $\mathrm{V})$.

3. Émile, livre V, OC IV, p. 837 .

PHILOSOPHIQUES 43/1 - Printemps 2016, p. 3-21 
reçoit une formulation spécifique ${ }^{4}$. Rousseau y déclare, non sans ironie: « $\mathrm{Si}$ j'étais prince ou législateur, je ne perdrais pas mon temps à dire ce qu'il faut faire; je le ferais, ou je me tairais ». Pour autant, il le précise aussitôt, ce n'est pas par défaut qu'il se résout à «écrire sur la Politique». Un motif précis l'y a déterminé: "Né citoyen d'un État libre, et membre du souverain, quelque faible influence que puisse avoir ma voix dans les affaires publiques, le droit d'y voter suffit pour m'imposer le devoir de m'en instruire». La rédaction du Contrat social a donc pour but de dégager les principes qui permettront au citoyen de s'orienter dans l'exercice de ses droits politiques. Or, en toute rigueur, pour comprendre ces principes, il faut que le citoyen sache ce qui lui importe. On peut considérer en un sens que l'ouvrage tout entier répond de façon diffuse à cette question. Elle est cependant abordée frontalement cette contribution voudrait le montrer - dans un passage aussi dense que bref, aussi décisif que problématique, du chapitre IX du livre III: Rousseau $\mathrm{y}$ trace les contours et les limites de ce qu'on pourrait appeler la politique $d u$ citoyen ${ }^{5}$.

Notre lecture de ce texte ne pourra s'appuyer, ni positivement ni négativement, sur celles qui en auraient déjà été proposées: loin d'avoir retenu l'attention des commentateurs (ils ont pourtant scruté le Contrat social jusque dans le détail de ses formulations), il est pour ainsi dire resté orphelin ${ }^{6}$. Après avoir examiné le contexte particulier dans lequel ce passage intervient (il explique en partie son occultation), nous l'analyserons de façon aussi précise que possible en restituant sa signification dans l'économie conceptuelle du Contrat social, pour enfin montrer qu'il pose un problème crucial pour la compréhension d'ensemble de la pensée politique de Rousseau.

\section{Un argument mineur dans un chapitre de transition?}

Le troisième livre du Contrat social, comme l'indique la table des matières de I762, traite "des lois politiques, c'est-à-dire, de la forme du Gouvernement ». Les chapitres I à VIII déterminent la fonction du gouvernement dans les institutions politiques (il est chargé de l'administration des lois), définissent ses trois formes simples (démocratie, aristocratie, monarchie) et montrent qu'ils peuvent donner lieu à une infinité de combinaisons (Rous-

4. On notera que les motifs qui conduisent Émile à s'instruire des principes du droit politique (choisir le lieu dans lequel il lui convient de vivre) différent de ceux, envisagés ici, qui poussent Rousseau à établir ces principes dans le Contrat social. Cette différence détermine la manière dont ils sont abordés dans ces deux œuvres.

5. Je remercie Christophe Litwin dont les remarques m’ont permis d'améliorer cet article sur plus d'un point.

6. On ne voit pas que des commentaires spécifiques lui aient été consacrés et, dans leur annotation, les nombreuses éditions du Contrat social restent quasiment muettes à son sujet (de rares et laconiques exceptions seront mentionnées). Ce constat de déshérence vaut pour ma propre annotation du Contrat social, G-F Flammarion, 200I, 20I22. Je renverrai, pour le commentaire, à cette édition désormais notée CS. 
seau renouvelle la théorie des gouvernements mixtes $\left.{ }^{7}\right)$. Les chapitres $\mathrm{X}$ à XVIII établissent que le gouvernement tend toujours à usurper la souveraineté du peuple, examinent les causes et les effets de cette dégénérescence et cherchent les moyens de la freiner (cette tâche sera poursuivie au livre IV).

Entre ces deux ensembles, le court chapitre IX s'intitule «Des signes d'un bon Gouvernement». Une affirmation liminaire tire la conclusion des chapitres précédents: il n'y a pas lieu de demander quelle est la forme du bon gouvernement, puisque chaque peuple a une forme spécifique qui lui convient. Cependant, on peut se demander s'il n'y a pas un critère qui permettrait de reconnaître qu'un peuple a trouvé la sienne. Un paragraphe conclusif répondra positivement en désignant un critère indirect (un signe) empiriquement vérifiable: la démographie. Selon que la population d'un peuple croît ou décroit, on peut conclure qu'il est bien ou mal gouverné. La thèse "populationniste" que soutient ce chapitre a été largement commentée, souvent d'ailleurs pour observer qu'elle est assez banale au XVIII ${ }^{e}$ siècle ${ }^{8}$. Michel Senellart en a proposé une réévaluation fine en montrant la modulation singulière qu'elle reçoit dans la pensée de Rousseau'. Mais avant de répondre ainsi à la question qu'il s'était initialement posée, Rousseau explique pourquoi elle reste en général irrésolue: les critères que l'on mobilise pour la trancher étant qualitatifs, ils donnent lieu à des évaluations divergentes et nourrissent autant de controverses. Dans la structure argumentative du chapitre, ce troisième paragraphe, pourtant le plus long, a donc une fonction purement négative: montrer que, la voie directe et qualitative étant aporétique, il faut en emprunter une autre, indirecte et quantifiable. On peut sans doute expliquer par là que les commentateurs du Contrat social ne lui aient accordé qu'une attention discrète. C'est précisément à ce paragraphe que mon propos sera consacré mais, pour la commodité de l'analyse, sa brièveté le permettant, je retranscris ce chapitre en entier ${ }^{10}$.

7. Sur cette question voir B. Bernardi, La fabrique des concepts, recherches sur l'invention conceptuelle chez Rousseau, Paris, Honoré Champion 2006, Partie I, chap. III: Gouvernements mixtes: un paradigme phénix.

8. Cette préoccupation, en effet ancienne, était perçue avec une acuité inédite depuis la révocation de l'Édit de Nantes et la dépopulation quantitative et qualitative de la France qu'elle avait entrainée. Voir, par exemple, ce qu'en dit Saint-Simon dans ses Mémoires, La Pléiade, vol. V, p. 552-555. Depuis le XVII e siècle, les enquêtes sur la natalité et la mortalité s'étaient développées en Angleterre et à Genève en particulier. Mais les premières grandes théories démographiques sont postérieures: les Recherches et considérations sur la population de la France de Jean-Baptiste Moheau datent de I778, et le grand ouvrage de Thomas Malthus de I798.

9. M. Senellart, "La population comme signe du bon gouvernement ", in Rousseau et la philosophie, A. Charrak et J. Salem dir., Publications de la Sorbonne, 2004, p. I89-2 I 2.

10. J'omets cependant une longue note ajoutée lors de l'impression du Contrat social sur laquelle je reviendrai. 


\section{6• Philosophiques / Printemps 2016}

\section{Des signes d'un bon Gouvernement}

Quand donc on demande absolument quel est le meilleur Gouvernement, on fait une question insoluble comme indéterminée; ou si l'on veut, elle a autant de bonnes solutions qu'il y a de combinaisons possibles dans les positions absolues et relatives des peuples.

Mais si l'on demandait à quel signe on peut connaître qu'un peuple donné est bien ou mal gouverné, ce serait autre chose, et la question de fait pourrait se résoudre.

Cependant on ne la résout point, parce que chacun veut la résoudre à sa manière. Les sujets vantent la tranquillité publique, les Citoyens la liberté des particuliers; l'un préfère la sûreté des possessions, et l'autre celle des personnes; l'un veut que le meilleur Gouvernement soit le plus sévère, l'autre soutient que c'est le plus doux; celui-ci veut qu'on punisse les crimes, et celui-là qu'on les prévienne; l'un trouve beau qu'on soit craint des voisins, l'autre aime mieux qu'on en soit ignoré; l'un est content quand l'argent circule, l'autre exige que le peuple ait du pain. Quand même on conviendrait sur ces points et d'autres semblables, en serait-on plus avancé? Les quantités morales manquant de mesure précise, fût-on d'accord sur le signe, comment l'être sur l'estimation?

Pour moi, je m'étonne toujours qu'on méconnaisse un signe aussi simple, ou qu'on ait la manvaise foi de n'en pas convenir. Quelle est la fin de l'association politique? C'est la conservation et la prospérité de ses membres. Et quel est le signe le plus sûr qu'ils se conservent et prospèrent? C'est leur nombre et leur population. N'allez donc pas chercher ailleurs ce signe si disputé. Toutes choses d'ailleurs égales, le Gouvernement sous lequel, sans moyens étrangers sans naturalisations sans colonies les Citoyens peuplent et multiplient davantage, est infailliblement le meilleur: celui sous lequel un peuple diminue et dépérit est le pire. Calculateurs, c'est maintenant votre affaire; comptez, mesurez, comparez.

\section{Question de fait et question de droit: de quoi le signe est-il le signe?}

La compréhension de ce texte demande que l'on revienne d'abord sur la définition que le chapitre I du livre III a donnée du gouvernement, " confondu mal à propos avec le Souverain». La souveraineté, considérée ici comme puissance législative, "appartient au peuple et ne peut appartenir qu'à lui ». C'est un des principes les plus fermes de Rousseau. Le gouvernement ne légifère pas, il administre : il est "chargé de l'exécution des lois, et du maintien de la liberté, tant civile que politique». Ce remaniement conceptuel majeur implique qu'il n'y a qu'une forme de souveraineté qu'on puisse qualifier de «bonne» ou, dans le vocabulaire de Rousseau, de «légitime»: la souveraineté du peuple ${ }^{11}$. La question traditionnelle du «bon gouvernement », puisqu'elle ne peut plus porter que sur la correspondance entre la

11. Voir CS II, VI, la célèbre affirmation: "Tout gouvernement légitime est républicain». Une note précise: "Je n'entends pas seulement par ce mot une Aristocratie ou une Démocratie, mais en général tout gouvernement guidé par la volonté générale, qui est la loi. 
forme du gouvernement et ce principe constitutif de la souveraineté, est donc d'emblée minorée. La première partie du livre III conduit de plus à sa relativisation. En effet, on l'a vu, chaque peuple a une forme de gouvernement qui lui convient, et cette convenance est déterminée par une multiplicité de critères qui sont déclinés par le chapitre VIII: l'importance et la densité de sa population, l'étendue de son territoire, sa géographie, sa fertilité, son climat. Cette thèse est bien entendu reprise de Montesquieu ${ }^{12}$.

Nous pouvons à partir de là mieux déterminer la question que Rousseau résoudra dans ce chapitre. L'augmentation ou la diminution de la population, va-t-il montrer, est le signe de ce qu'un peuple «donné » est bien ou mal gouverné, et ce signe, parce qu'il est quantifiable, permet d'opérer une comparaison entre des termes par ailleurs incommensurables puisque chaque peuple à une forme de gouvernement particulière. Il est d'ailleurs intéressant de noter que le Contrat social ne retient ici que le critère de l'augmentation ou diminution de la population, alors que le développement parallèle et plus développé que l'Émile consacre à cette question insiste plutôt sur la répartition de la population et fait de l'importance relative de la ville capitale le critère le plus pertinent de ce qu'un pays est bien ou mal gouverné ${ }^{13}$. Mais le critère le plus aisément mesurable est privilégié ici, et c'est pourquoi ce chapitre peut se conclure par une interpellation en large part ironique: "Calculateurs, c'est maintenant votre affaire; comptez, mesurez, comparez $»^{14}$. Cependant, pour identifier l'objet de cette démonstration, il faut distinguer le signe de la cause: le signe est un symptôme de bonne ou de mauvaise santé, pas ce qui l'explique ${ }^{15}$. L'accroissement de sa population dit qu'un peuple «prospère» mais ne dit rien de ce qui explique cette prospérité. Il ne faut pas non plus se tromper sur ce que montre cette comparaison: elle ne permet pas d'affirmer qu'un peuple dont la démographie est florissante a un

Pour être légitime il ne faut pas que le Gouvernement se confonde avec le Souverain, mais qu'il en soit le ministre: alors la monarchie elle-même est république».

12. L'incipit de CS III, VIII est explicite: «La liberté n'étant pas un fruit de tous les Climats n'est pas à la portée de tous les peuples. Plus on médite ce principe établi par Montesquieu, plus on en sent la vérité ".

13. Émile, L. V, OC IV p. 85 I-852: «La seconde marque de la bonté relative du gouvernement et des lois se tire aussi de la population, mais d'une autre manière, c'est-à-dire de sa distribution, et non pas de sa quantité ». Le Contrat social aborde sous un autre angle (III. XIII) la question des capitales et la répartition de la population.

14. Les Essais d'arithmétique politique de William Petty, publiés à titre posthume en I690 n'ont été traduits qu'en I757 mais ils étaient indirectement connus. L'application des mathématiques à la démographie est discutée au milieu du XVIII ${ }^{\mathrm{Ie}}$, notamment par l'Essai sur les éléments de philosophie de D’Alembert (I759).

15. L'Émile, parlant de la dépopulation, use de ce vocabulaire médical p. $85 \mathrm{I}$ : «C'était la politique du bon abbé de Saint-Pierre de chercher toujours un petit remède à chaque mal particulier, au lieu de remonter à leur source commune, et de voir qu'on ne les pouvait guérir que tous à la fois. Il ne s'agit pas de traiter séparément chaque ulcère qui vient sur le corps d'un malade, mais d'épurer la masse du sang qui les produit tous ». 


\section{8• Philosophiques / Printemps 2016}

meilleur gouvernement que celui dont la démographie est en berne, mais seulement que la forme de son gouvernement convient mieux au premier peuple que la sienne au second. C'est une plus ou moins grande convenance que l'on mesure. D'où la condition mise à la comparaison: "Toutes choses d'ailleurs égales». La question résolue est donc de savoir si "un peuple donné » a le gouvernement qui lui convient. Rousseau la qualifie de "question de fait».

Pour autant, une question de fait renvoie toujours à une question de droit, au sens axiologique du terme: elle relève d'une "estimation", nécessairement qualitative, qui dépend des critères que l'on a préalablement retenus. Cette remarque doit nous alerter sur la façon dont il faut lire ce chapitre: il est en effet difficilement concevable que Rousseau écarte en principe la question de droit pour ne retenir que la question de fait et disqualifie les "quantités morales" pour ne considérer que des données empiriques ${ }^{16}$. Une telle démarche serait évidemment contradictoire avec l'orientation générale de sa pensée qui entend toujours "examiner les faits par le droit $»^{17}$. Le sens de cette règle est précisé au livre $\mathrm{V}$ de l'Émile dans lequel est définie la "science du droit politique ${ }^{18}$. Celle-ci ne saurait se contenter d'étudier «le droit positif des gouvernements établis » (cette critique s'adresse à Montesquieu). Elle doit au préalable former les "principes du droit politique»: "il faut savoir ce qui doit être pour bien juger de ce qui est». Un peu plus loin, Rousseau renchérit: "Avant d'observer, il faut se faire des règles pour ses observations: il faut se faire une échelle pour y rapporter les mesures qu'on prend. Nos principes de droit politique sont cette échelle». On peut difficilement imaginer qu'une thèse aussi centrale soit reniée dans l'ouvrage dont l'Émile présente ainsi le résumé et qui porte précisément pour sous-titre Principes $d u$ droit politique. Nous devons donc comprendre que ce n'est pas une raison principielle mais méthodologique et circonstancielle qui conduit Rousseau à se déplacer, au cours de ce chapitre, de la question de droit vers la question de fait. Le critère démographique a pour seul mérite, mais il n'est pas mince, de couper court à la discussion sur les principes axiologiques d'une évaluation. Ses lecteurs ont si bien suivi la logique de ce déplacement qu'ils semblent n'avoir reconnu de fonction que rhétorique à la longue suite d'oppositions évoquées dans le passage qui nous occupe ici. Pour autant, que leur discussion soit écartée dans ce chapitre ne peut nous dispenser d'examiner les questions soulevées et d'identifier le principe des évaluations divergentes qu'elles suscitent.

16. Nous reviendrons plus loin sur l'expression «quantités morales» et les perspectives auxquelles elle ouvre.

17. Discours sur l'origine et les fondements de l'inégalité parmi les hommes (désormais DOI), OC III, p. I 82 .

18. Émile, L. V, p. 836-837. 


\section{Tranquillité publique et liberté des particuliers: deux critères du bon gouvernement.}

Les verbes employés par Rousseau dans ce passage ne laissent pas de doute: vanter, préférer, vouloir, trouver beau, aimer, être content, exiger, estimer, nous avons affaire à une série d'appréciations. La connotation affective ou esthétique de ces verbes est-elle de nature à dégrader le statut des jugements qu'ils supportent, à en faire l'expression de préférences subjectives, au mieux de jugements de goût, plutôt que de véritables jugements de valeur? Faut-il déduire de la façon dont ces oppositions sont introduites ( «chacun veut la résoudre à sa manière ») que ce sont là autant de réponses personnelles à la question du bon gouvernement ? On peut objecter à cette lecture, en premier lieu, que l'opposition entre valeurs et affects est étrangère à la pensée politique de Rousseau, comme en atteste la place qu'il accorde à "l'amour des lois », «de la liberté » ou "de la patrie». On pourrait aussi attirer l'attention sur le fait que, dans sa pensée politique, le jugement de goût a un statut précis en relation avec la problématique des mœurs et de l'opinion publique ${ }^{19}$. Mais développer ces arguments nous éloignerait de notre objet. Au demeurant, il suffit pour écarter cette interprétation d'examiner le premier conflit axiologique évoqué par notre texte: "Les sujets vantent la tranquillité publique, les Citoyens la liberté des particuliers».

On notera en premier lieu que ce n'est pas tel ou tel individu singulier, ni telle ou telle catégorie particulière que cet énoncé considère mais bien, dans leur généralité, "les sujets» et «les citoyens». Nous avons ici affaire aux concepts fondamentaux qui définissent le double rapport sous lequel on doit considérer les membres du corps politique ${ }^{20}$. Le chapitre VI du livre I les a nettement définis: "À l'égard des associés ils prennent collectivement le nom de peuple, et s'appellent en particulier Citoyens comme participants à l'autorité souveraine, et Sujets comme soumis aux lois de l'État». Le point de vue des citoyens pris «en particulier» (il est corollaire mais distinct de celui qu'ils ont collectivement comme peuple) est celui, actif, de membres du souverain: ils ont pour objet le bien commun, leur volonté commune est pour cette raison qualifiée de générale et s'exprime par la loi. Le point de vue des sujets est celui, passif, des membres de l'État, pour qui l'obéissance à la loi est un devoir mais aussi une contrainte qui peut leur être imposée. Cette différence de point de vue donne sa nécessité et sa légitimité à la coercition,

19. Les textes décisifs sur ces questions sont, dans le Contrat social, le chapitre VII du livre IV, les analyses de la Lettre à d'Alembert, et le chapitre II des Considérations sur le gouvernement de Pologne. Voir, B. Bernardi, «Rousseau et la généalogie du concept d'opinion publique", in Jean-Jacques Rousseau en 2012, Puisqu'enfin mon nom doit vivre, Michael O'Dea dir., SVEC, Oxford, Voltaire Foundation, 20I 2, p. 95-I 27.

20. Georges Beaulavon, libelle ainsi l'unique note consacrée à ce chapitre par son édition du Contrat social, Société nouvelle de librairie et d'édition, Paris, I903, p. 244: "Les sujets, dans une monarchie; les citoyens dans une démocratie». Cette identification est bien évidemment impossible dans la terminologie de Rousseau. 
comme le montre le chapitre VII du même livre: «le souverain n'étant formé que des particuliers qui le composent n'a ni ne peut avoir d'intérêt contraire au leur [....]. Mais il n'en est pas ainsi des sujets envers le Souverain, auquel malgré l'intérêt commun, rien ne répondrait de leurs engagements s'il ne trouvait des moyens de s'assurer de leur fidélité ». Si les particuliers membres du corps politique, comme citoyens, généralisent leur volonté en considérant l'intérêt commun, ils sont enclins, comme sujets, à privilégier leur intérêt privé. C'est à l'aune de ces deux caractérisations qu'il faut expliquer l'affirmation selon laquelle «les sujets vantent la tranquillité publique, les Citoyens la liberté des particuliers".

Pourquoi les sujets vantent-ils la tranquillité publique? Parce qu'ils attendent de l'État d'être protégés de la menace qu'autrui représente pour eux et de pouvoir conduire leurs affaires privées en sécurité. Un bon gouvernement, de leur point de vue, est celui qui les place sous l'égide de la puissance publique. Le second Discours n'avait pas donné d'autre origine réelle ${ }^{21}$ à l'organisation politique de la société: pour sortir de «l'horrible état de guerre » qui suit l'institution de la propriété, les riches persuadent les pauvres qu'ils doivent tous se soumettre à un pouvoir commun ${ }^{22}$. Ainsi est engagé le processus par lequel le peuple consent «à laisser augmenter sa servitude pour affermir sa tranquillité ${ }^{23}$. Les sujets sont prêts à voir restreindre leur liberté pour garantir leur sécurité. Pourquoi les Citoyens vantent-ils la liberté des particuliers? Précisément parce qu'ils n'admettent pas les termes de cet échange de la liberté contre la sécurité (Rousseau dit toujours "sûreté»). Ils ne prétendent pas rester indépendants (dès lors que l'on est entré dans l'état civil l'indépendance naturelle disparait) mais, en se soumettant à la seule autorité de la loi, ils exigent d'être affranchis de toute «dépendance personnelle». La liberté que revendiquent les citoyens est donc double. Elle est la liberté politique qui assure la participation aux décisions collectives en quoi consiste l'exercice de la souveraineté. Elle est aussi la liberté civile qui consiste à n'être limité dans ses actions que par la volonté générale, jamais par celle d'un particulier: dans un ordre politique légitime aucun citoyen n'est sous la dépendance d'un autre ${ }^{24}$. On notera que la liberté civile ainsi entendue est la condition de la liberté politique et que c'est pour

21. Cette qualification doit s'entendre au sens d'effective mais renvoie aussi à la distinction du réel et du personnel. Chez Rousseau cette seconde acception correspond à une thèse essentielle: les rapports réels (ceux qui ont pour objet la possession des choses) sont au fondement de la société civile et de la guerre qui en est le corollaire. Voir CS I, IV : "C'est le rapport des choses et non des hommes qui constitue la guerre, et l'état de guerre ne pouvant naître des simples relations personnelles, mais seulement des relations réelles, la guerre privée ou d'homme à homme ne peut exister, ni dans l'état de nature où il n'y a point de propriété constante, ni dans l'état social où tout est sous l'autorité des lois".

22. DOI, p. I76-I79.

23. Ibid., p. I 87 .

24. CS I, VIII. 
cette raison qu'une certaine égalité de fortune et de condition doit être assurée $^{25}$. La protection de la liberté politique et civile est donc la priorité du peuple (le corps politique pris collectivement) mais aussi des citoyens pris en particulier. La qualification en chiasme (tranquillité publique vs liberté des particuliers) a pour objet de montrer que la tranquillité, pour les sujets, est l'affaire du gouvernement tandis que la liberté, pour les citoyens, est leur propre affaire. Les sujets attendent du gouvernement qu'il assure leur tranquillité, les citoyens qu'il respecte et maintienne leur liberté. On peut déjà observer (nous y reviendrons) que Rousseau, dès le début du livre III, a fixé pour tâche au gouvernement non seulement «l'exécution des lois» mais aussi «le maintien de la liberté, tant civile que politique » et que cette seconde détermination correspond au point de vue dont nous voyons ici qu'il est celui des citoyens.

\section{La politique du sujet et celle du citoyen: une alternative axiologique.}

Nous avons clarifié le statut de cette première opposition: loin d'être arbitraire, elle renvoie à la dualité des positions réelles de sujet et de citoyen dont participent nécessairement les membres du corps politique. Nous avons aussi déterminé, au moins sommairement, les critères d'évaluation dont ces positions sont respectivement porteuses: la paix civile d'un côté, la liberté de l'autre. C'est le «double rapport» sous lequel «chaque individu, contractant, pour ainsi dire avec lui-même se trouve engagé " qui génère ce conflit de valeurs ${ }^{26}$. Cela posé, il semble possible de trancher l'alternative interprétative qui commande la lecture de ce texte: doit-on y voir l'énumération d'une série de six oppositions portant sur des questions distinctes et faisant intervenir des interlocuteurs différents, ou bien y reconnaitre la déclinaison d'une même opposition matricielle, celle des sujets et des citoyens? Implicitement, la première lecture semble avoir dominé ${ }^{27}$. Pourtant, d'un point de vue rhétorique, on peut observer en faveur de la seconde que nous avons affaire à une seule et même phrase, formée par l'apposition de six couples d'énoncés adversatifs, sans aucune subordination: les sujets grammaticaux récurrents des deux séries d'énoncés «l'un, celui-ci », «l'autre, celui-là », ont

25. CS II, XI.

26. CS I, 7 .

27. Une exception est à relever : Jean-Louis Lecercle, dans son édition du Contrat social (Editions sociales, Paris, I955, p. I47), place ici une note démarquée de celle de Beaulavon et sujette à la même objection, mais qui discerne la continuité de cette série: «Dans ce parallèle, «l'un» est toujours le sujet (d'une monarchie), «l'autre» le citoyen (d'une démocratie». Du silence des autres éditeurs, on ne peut rien conclure avec certitude. Mais l'examen de diverses traductions anglaises (M. Cronston, I968; J. Masters, I978; J. Conway, I987; V. Gourevitch, I997; J. Scott, 20I2; Q. Hoare, 20I2) montre que la première lecture est dominante. Cela est particulièrement net dans la dernière en date (Quintin Hoare, Penguin, 20I2), qui écrit «one man ", "an other ", "this man" etc. Seuls Cronston et Conway font clairement la lecture que je défends ici. 
donc logiquement pour antécédents ceux, nominativement désignés, de l'opposition initiale: «les sujets» et "les citoyens». Il faut cependant, pour soutenir cette lecture, concéder à Rousseau la licence grammaticale qui consiste à passer d'un pluriel collectif dans le premier couple d'énoncés au singulier distributif dans les cinq suivants. Au demeurant, c'est conceptuellement qu'il faut montrer que nous assistons au déploiement de deux séries alternatives de jugements qui ont chacune leur propre systématicité. Avant d'appuyer cette compréhension par l'analyse, on peut la suggérer intuitivement en présentant la rédaction de ce passage, sans rien en retrancher, dans un tableau:

Les sujets vantent la tranquillité publique l'un préfère la sûreté des possessions

l'un veut que le meilleur Gouvernement soit le plus sévère

celui-ci veut qu'on punisse les crimes

l'un trouve beau qu'on soit craint des voisins l'un est content quand l'argent circule les Citoyens la liberté des particuliers et l'autre celle des personnes

l'autre soutient que c'est le plus doux et celui-là qu'on les prévienne

l'autre aime mieux qu'on en soit ignoré l'autre exige que le peuple ait du pain

Commençons par lire, l'une à la suite de l'autre, les deux colonnes ainsi formées. La valorisation par les sujets de la tranquillité publique une fois posée, les cinq propositions suivantes en déclinent les tenants et les aboutissants. Parce qu'ils ont d'abord en vue leur intérêt privé, les sujets tiennent par dessus tout à la protection de leurs biens. Nous retrouvons la généalogie de l'institution politique établie par le second Discours: les relations réelles déterminent les relations personnelles et le but de la société politique est de garantir à chacun ses possessions. La sévérité du gouvernement est sa condition première et s'exprime dans sa fonction répressive ou punitive. Pour le sujet, autrui représente d'abord une menace et, pour autant que cela dépend de lui, il s'efforce d'y répondre en inspirant de la crainte à ses voisins ${ }^{28}$. D'un autre côté, parce qu'il est avant tout intéressé par la possession et l'accroissement de ses richesses, sa conception de l'économie sera mercantiliste: la circulation de l'argent (et des marchandises) est le gage d'une économie profitable. Il en va tout autrement du citoyen. La liberté étant pour lui le premier bien, il attendra toute autre chose du gouvernement. Les relations personnelles primant sur les relations réelles, la sûreté des personnes lui importe plus que celle des biens parce que c'est de la première que dépendent la liberté politique (pouvoir se prononcer en toute indépendance sur les affaires communes) et la liberté civile (pouvoir conduire son existence sans être soumis à l'autorité ou subordonné à la puissance de quiconque). Le bon gouvernement ne se signalera pas par sa fermeté répressive (la répression est l'aveu d'un échec de la politique) mais par sa capacité 
à prévenir les infractions à la loi : il doit s'efforcer pour cela de la faire aimer plutôt que de la faire craindre ${ }^{29}$. C'est, pour Rousseau, une thèse aussi centrale en politique qu'en théologie: le premier attribut de la puissance est la bonté $^{30}$. Un gouvernement plus «doux» n'est pas un gouvernement dont l'autorité est moindre mais celui dont l'autorité repose sur la persuasion plutôt que sur la contrainte: en exerçant le soft power qui consiste à former les mœurs par l'opinion, le gouvernement se fait l'héritier du législateur ${ }^{31}$. Dans les rapports avec ses voisins, le citoyen cherche avant tout à préserver son autonomie qui est l'analogue, dans l'état civil, de l'indépendance naturelle. Il vaut mieux à cet égard une absence de rapports que des rapports d'hostilité. Quant à sa conception de la responsabilité économique du gouvernement, elle répond à un impératif de justice sociale (que chacun ait les moyens de son existence) et d'égalité politique ( "que nul ne soit assez pauvre pour être contraint de se vendre ${ }^{32}$ ). Au nom de ce double impératif, il "exige» (ce terme a une forte résonance politique) "que le peuple ait du pain ". On le reconnaitra sans doute après cette lecture cursive, Rousseau oppose deux horizons axiologiques respectivement enracinés dans la position du sujet et celle du citoyen et orientés par deux valeurs matricielles: la sécurité et la liberté.

Pour autant, à relire ce tableau ligne par ligne, certaines formulations peuvent susciter quelques doutes sur l'opposition de ces deux conceptions. La première de ces difficultés a été déjà résolue. En effet, nous avons vu pourquoi, en dépit de ce que l'on pourrait attendre, ce n'est pas la liberté du peuple mais celle "des particuliers» que "vantent» les citoyens: Rousseau respecte jusque dans les termes employés la distinction qu'il a établie au livre I entre le souverain considéré collectivement (c'est le peuple) et distributivement (ce sont les citoyens) ${ }^{33}$. Il parait de prime abord plus difficile d'expliquer pourquoi les sujets n'auraient pas souci de la sûreté des personnes, et respectivement les citoyens de celle des biens. Aussi bien, notre texte ne dit rien de tel. Il observe seulement une préférence: les sujets donnent la priorité à la sûreté des biens, les citoyens à celle des personnes. Sans doute faut-il comprendre qu'en cas de conflit entre ces deux exigences, les sujets privilé-

29. Rappelons que Rousseau, CS II, XII, ne fait que mentionner les «lois criminelles» qui «dans le fond sont moins une espèce particulière de lois, que la sanction de toutes les autres». Contrairement au républicanisme classique, le droit de punir n'a chez lui qu'une place minime. C'est le devoir de prévenir qui lui importe.

30. Depuis le XVII siècle cette corrélation est en débat: B. Bernardi, Le principe d'obligation: sur une aporie de la modernité politique, EHESS/Vrin, 2007, chap. 4. La querelle de l'obligation: Pufendorf, Leibniz et Barbeyrac.

31. Cette question distingue également Rousseau du républicanisme classique. Sur ce point, B. Bernardi, «Rousseau et la généalogie du concept d'opinion publique », art. cit.

32. CS II, XI.

33. L'engagement que le citoyen prend «comme membre du Souverain envers les particuliers» (CS I, VII) est en premier lieu de défendre leur liberté. 
gieront la première, les citoyens la seconde. Mais cette relation de préférence, au delà de cet item particulier, ne vaut-elle pas pour l'ensemble des oppositions évoquées? Rousseau ne dit en effet à aucun moment que les sujets ne font pas leurs les valeurs portées par les citoyens, ni réciproquement les citoyens celles que soutiennent les sujets, mais que l'ordre préférentiel qu'ils adoptent est inversé. Il ne dit pas en particulier que le citoyen veut être ignoré de ses voisins mais qu'il «aime mieux» en être ignoré qu'en être craint.

Cette dernière proposition soulève cependant une difficulté d'une autre nature ${ }^{34}$. Concerne-t-elle, comme les précédentes, l'ordre intérieur de la société (les relations des particuliers entre eux) ou plutôt les rapports des sociétés entre elles? Les autres emplois de la notion de voisinage dans le Contrat social renvoient à la seconde compréhension: les voisins y sont chaque fois les peuples voisins ${ }^{35}$. Nous pouvons donc lire ici l'opposition de deux conceptions de ce que doit être la politique étrangère d'un bon gouvernement: pour le sujet il doit affirmer sa puissance face à celle des autres, pour le citoyen il doit défendre l'indépendance du peuple en se tenant à leur écart $^{36}$. Cette thèse a d'ailleurs un corollaire important: Rousseau n'envisage la politique militaire d'un "gouvernement légitime» que sur le mode défensif. Mais faut-il écarter la première compréhension de cette proposition? Il y a de bonnes raisons d'en douter.

Le voisinage, sous la plume de Rousseau, désigne toujours un rapport inscrit dans un espace entendu comme objet d'appropriation et qui, pour cela même, est source d'hostilité et de guerre ${ }^{37}$. Or, sous ce concept, il enveloppe aussi bien les rapports des particuliers entre eux que celui des corps politiques. Le Discours sur l'économie politique le disait vertement: celui qui a «un voisin puissant» est «un homme perdu» ${ }^{38}$. Ce danger menace particulièrement l'agriculteur dont la subsistance l'attache à la terre, et c'est pourquoi Émile sera artisan ou ouvrier plutôt qu'agriculteur: "L'ennemi, le prince, un voisin puissant, un procès peut lui enlever [son] champ $»^{39}$. Le Contrat social, reprenant une thèse centrale des Principes $d u$ droit de la guerre, constate de son côté que «tous les peuples ont une espèce de force centrifuge, par laquelle ils agissent continuellement les uns contre les autres

34. Les observations faites sur un premier état de ce texte par Christophe Litwin et, particulièrement sur ce point par Dominique Horvilleur et Louis Guerpillon m'ont aidé à en approfondir l'examen.

35. Ces occurrences se trouvent aux chapitres VIII, IX et X du livre II, VI du livre III et VIII du livre IV.

36. C'est le conseil de Rousseau aux Corses: protéger sa liberté en restant ignorés de ses voisins: OC III, p. 903.

37. Les relations personnelles peuvent susciter une agressivité ponctuelle, seules les relations réelles peuvent créer un état de guerre. CS I, IV.

38. Discours sur l'économie politique, OC III, p. 272.

39. Émile, livre III, OC IV, p. 470 
et tendent à s'agrandir aux dépens de leurs voisins, comme les tourbillons de Descartes ${ }^{40}$. Pour les particuliers comme pour les États, les rapports de voisinage sont des rapports de puissance. Déjà, le second Discours avait établi la corrélation entre ces deux figures. L'appropriation de la terre par les particuliers a engendré "l'horrible état de guerre» auquel «le riche» imagine d'échapper en convainquant «ses voisins" de s'unir pour "assurer à chacun la possession de ce qui lui appartient $»^{41}$. Les corps politiques se sont alors constitués différemment suivant que «l'Administration suprême » était inégale (monarchie et aristocratie) ou gardée «en commun» (démocratie): "Les uns restèrent uniquement soumis aux Lois, les autres obéirent bientôt à des Maîtres. Les Citoyens voulurent garder leur liberté, les sujets ne songèrent qu'à l'ôter à leurs voisins, ne pouvant souffrir que d'autres jouissent d'un bien dont ils ne jouissaient plus eux-mêmes. En un mot, d'un côté furent les richesses et les Conquêtes, et de l'autre le bonheur et la vertu ${ }^{42}$. Ces textes s'entrexpliquent: c'est en vertu d'un principe également valable en droit politique externe et interne que le citoyen aime mieux être "ignoré des voisins » qu'en être craint.

Il n'en reste pas moins que ce repli sur soi peut paraître contradictoire avec la représentation que l'on se fait communément de la citoyenneté comme implication dans une existence collective. Cette question est assez essentielle pour la pensée de Rousseau pour qu'on s'y attarde un peu. L'implication que leur citoyenneté requiert des membres du corps politique a un lieu privilégié ou plus exactement nécessaire: l'assemblée du peuple. La participation aux délibérations collectives est la seule façon d'exercer réellement sa liberté politique. Ce principe explique les thèses les plus polémiques du troisième livre du Contrat social: que «le Gouvernement Démocratique convient aux petits États» (chapitre III), que «le Souverain ne saurait agir que quand le peuple est assemblé » (chapitre XII), enfin «qu'à l'instant qu'un Peuple se donne des Représentants, il n'est plus libre» (chapitre XIV). On a cru pouvoir tirer de ces thèses l'idée selon laquelle l'existence privée du sujet devrait se résorber dans l'existence publique du citoyen ${ }^{43}$. Une idée que l'on pourrait d'ailleurs lire en toutes lettres dans ce même chapitre XIV: «Mieux l'État est constitué, plus les affaires publiques l'emportent sur les privées dans l'esprit des Citoyens. Il y a même beaucoup moins d'affaires privées, parce que la somme du bonheur commun fournissant une portion plus considérable à celui de chaque individu, il lui en reste moins à chercher dans les soins particuliers ". Or, à y regarder de plus près, ce texte prouve

40. CS II, IX. Principes $d u$ droit de la guerre, édition établie et présentée par B. Bernardi et G. Silvestrini, commentaire sous la dir. de B. Bachofen et C. Spector, Paris, Vrin, Textes et Commentaires, 2008, p. 52-55.

41. Discours sur l'origine et le fondement... OC III, p. I77.

42. Ibid. p. I86.

43. Cette lecture, depuis Benjamin Constant, a été défendue par tous ceux qui, avec plus ou moins de précautions, ont cru discerner chez Rousseau une tendance totalitaire. 
l'inverse de ce qu'on lui fait dire: Rousseau, une fois encore, raisonne en termes de proportion. Il formule même une fonction: mieux l'État est constitué, plus le bonheur personnel dépend des décisions collectives. Mais une fonction repose sur la distinction des termes qu'elle met en relation, ici le public et le privé. Si un des termes disparait, la proportion est détruite. La réduction du privé au public est donc préjudiciable, autant que celle du public au privé. Le chapitre IV du livre II, Des bornes du pouvoir souverain, avait précisément pour objet de montrer les valeurs limites que doit respecter cette fonction. Il faut pour cela «bien distinguer les droits respectifs des Citoyens et du Souverain, et les devoirs qu'ont à remplir les premiers en qualité de sujets, du droit naturel dont ils doivent jouir en qualité d'hommes ". Le statut du citoyen n'est concevable que si l'on considère "outre la personne publique... les personnes privées qui la composent». Il y a d'ailleurs tout lieu de penser qu'aux yeux de Rousseau la dépolitisation de la vie publique a pour corollaire la dissolution de l'intimité indispensable à la vie personnelle. Les mondanités prennent la place à la fois de la politique et de l'amitié ${ }^{44}$. Régler nos relations avec autrui sur notre position de sujets conduit alternativement à séduire et à intimider: deux rapports également ruineux pour ceux que nous devrions avoir comme hommes et comme citoyens.

L'analyse que nous venons de conduire a permis de montrer que Rousseau, dans ce passage, s'emploie méthodiquement à définir ce qui importe au citoyen, et que, pour ce faire, il mobilise les concepts fondamentaux de sa pensée politique et les idées maitresses de son anthropologie. Cohérent en lui même, ce passage l'est aussi avec l'économie conceptuelle du Contrat social. Mais ce double constat jette une lumière crue sur le paradoxe que représente ce chapitre dans sa structure d'ensemble: alors qu'il vient de distinguer clairement les priorités du sujet et celles du citoyen, alors qu'il donne à entendre que les secondes coïncident avec les "principes du droit politique ", Rousseau renonce à en faire les signes de ce qu'un peuple est bien gouverné.

\section{Les signes et les causes: Rousseau joue-t-il double jeu?}

De prime abord le paradoxe que nous venons de relever trouve son explication immédiate dans un argument qui, nous l'avons suggéré, est de nature épistémologique: "Les quantités morales manquant de mesure précise, fût-on d'accord sur le signe, comment l'être sur l'estimation?». Nous devons cependant examiner cet argument de plus près et, pour commencer, nous demander ce que sont les "quantités morales» dont parle Rousseau. Cette expression apparaît à deux reprises sous sa plume, toutes deux au livre III du Contrat social (la première au chapitre I, la seconde au chapitre IX) et,

44. C'est le sens de la critique de la "civilité " mal entendue dans le commentaire que Rousseau fait du Misanthrope de Molière dans sa Lettre à D’Alembert. 
dans les deux cas, pour exprimer la même idée. Il sera éclairant de les rapprocher.

Le premier chapitre du livre III, on l'a dit, traite $D u$ gouvernement en général. Rousseau définit le gouvernement (qu'il appelle aussi «prince») comme un «corps intermédiaire» qui établit le rapport entre le Souverain et l'État (soit entre les citoyens et les sujets). Pour que ce rapport soit équilibré, il faut que la puissance législative qu'exercent les citoyens et la puissance exécutive qui s'exerce sur les sujets soient proportionnées. La proportion est détruite, aux deux extrêmes, par l'anarchie (quand la puissance exécutive est nulle) et par la tyrannie (quand la puissance législative est anéantie). Pour figurer cette proportion, Rousseau fait appel au critère de population: «Pour tâcher de donner une idée des divers rapports qui peuvent régner entre ces deux extrêmes, je prendrai pour exemple le nombre du peuple, comme un rapport plus facile à exprimer ». Il étudie alors, avec force calculs, l'influence des variations de la population (l'ensemble des sujets) sur les deux autres termes (le souverain et le prince) et sur leurs rapports, soit la liberté du peuple et l'autorité du gouvernement. Prévoyant que l'on tournera en ridicule la "proportion continue " qu'il établit entre ces trois termes, il s'empresse de préciser: "si, pour m'exprimer en moins de paroles, j'emprunte un moment des termes de géométrie, je n'ignore pas, cependant, que la précision géométrique n'a point lieu dans les quantités morales". On comprendra mieux le choix de cette expression en relevant qu'elle est directement empruntée à Pufendorf ${ }^{45}$. Celui-ci, dans la discussion qu'il mène avec Grotius sur "la certitude des sciences morales", distingue les "quantités physiques» des "quantités morales », les premières étant susceptibles de «la dernière exactitude " tandis que les secondes ont toujours "quelque étendue » (Barbeyrac traduit ainsi le terme latin «latitudo»). Mais, de cette différence somme toute banale, Pufendorf donne une explication qui ne l'est pas: les quantités physiques «sont quelque chose de naturel, et par conséquent de fixe et d'invariable ", tandis que «les quantités morales doivent leur origine à l'institution et à l'estimation des Étres libres, dont l'intelligence et le jugement ne sont pas susceptibles d'une mesure physique». Plus déterminante encore est l'idée selon laquelle ces quantités concernent les «Êtres moraux». Or, pour Pufendorf, un être moral est celui qui «doit son origine à une institution humaine $»^{46}$. Pour Rousseau, il ne fait pas de doute que le peuple (souverain), le prince (le gouvernement) et le peuple (sujet) sont des êtres moraux. Mais cela est aussi vrai des sujets et des citoyens: ces deux statuts sont l'un et l'autre les produits de l'institution et par conséquent aussi des principes d'estimation (on peut dire des valeurs) dont ils sont porteurs: la liberté et la sécurité. Pour lui, comme pour Pufendorf, ce qui explique qu'on

45. Samuel Pufendrof, Du droit de la nature et des gens, Livre I, chap. II, $\mathbb{S}$ IX et X, trad. J. Barbeyrac, édition de Bâle, I732, p. 34-35.

46. Ibid., Livre VIII, chap. XII, $\mathbb{V}$ VII. 
puisse qualifier ces termes de «quantités ", c'est qu'ils sont corrélatifs: ils ne se définissent pas indépendamment l'un de l'autre mais par les rapports qu'ils ont entre eux. La «proportion continue » établie par le chapitre I avait pour objet de le montrer: "on ne saurait altérer aucun des trois termes sans rompre à l'instant la proportion ». Cependant, pour que les altérations de quantités soient précisément mesurables, il faut qu'elles puissent prendre une valeur numérique, ce qui n'est pas toujours le cas. C'est pour cette raison, dans les deux occurrences considérées, que Rousseau se tourne vers la population dont la quantité est mesurable: elle est un «exemple» dans le premier cas, un «signe» dans le second. Dans le passage du chapitre IX qui nous occupe, la difficulté soulevée ne porte donc pas sur le caractère prétendument arbitraire des critères (on peut envisager un "accord sur le signe») mais sur sa mesure. Rousseau se livre ainsi dans ce passage à une double opération de minoration. Il dégage une opposition conceptuelle majeure (entre ce qui importe au citoyen et au sujet), mais lui dénie toute pertinence comme signe du bon gouvernement. Dans le même mouvement, il restreint à une simple commodité de calcul celle du signe qu'il lui substitue (la population) pour cette seule raison qu'il est propre à la mesure.

Avoir mis ce point en évidence doit nous alerter sur le second retournement dont ce chapitre semble être l'objet: la longue note que Rousseau lui a ajoutée (elle est sensiblement plus étendue que le texte du chapitre) peut en effet être lue comme une correction majeure de sa signification. Cette note oppose les signes apparents d'un bon gouvernement et les conditions réelles de la prospérité d'un peuple. Le premier des signes trompeurs que Rousseau évoque renvoie à la thèse centrale de son premier Discours: les «lettres et les arts » peuvent fort bien prospérer alors que le peuple est dans la servitude et la misère. Le second, plus longuement développé, nous importe plus directement: "Il faut moins regarder au repos apparent, et à la tranquillité des chefs, qu'au bien-être des nations entières et surtout des états les plus nombreux ». Il y a une fausse tranquillité, celle de la servitude ${ }^{47}$ et une autre, bien différente, qui tient à la vitalité de l'esprit citoyen. Pour caractériser cette dernière, Rousseau paraphrase la préface des Histoires florentines : «Il semblait, dit Machiavel, qu'au milieu des meurtres, des proscriptions, des guerres civiles, notre République en devînt plus puissante; la vertu de ses citoyens, leurs mœurs, leur indépendance avaient plus d'effet pour la renforcer, que toutes ses dissensions n'en avaient pour l'affaiblir ». Et Rousseau de conclure par une thèse qui est sienne: «Un peu d'agitation donne du ressort aux âmes, et ce qui fait vraiment prospérer l'espèce est moins la paix que la liberté». À suivre rigoureusement la logique de cette phrase, il faudrait rectifier la conclusion du chapitre et dire que la démographie ne vaut que comme signe de la plus ou moins grande prospérité d'un peuple dont la

47. On se rappellera la formule cinglante de CS I, V: "On vit tranquille aussi dans les cachots, en est-ce assez pour s'y trouver bien ». 
cause véritable ne peut être que la liberté. Ce qui revient à faire de la politique du citoyen le critère de "ce qui doit être » au nom duquel on doit juger de "ce qui est». Au fond, nous devons lire la note ajoutée en fin de chapitre une sorte de contrepoids qui doit empêcher le lecteur attentif de confondre les signes avec les causes. Cette explication, pour exacte qu'elle soit, est-elle pleinement satisfaisante? Pourquoi Rousseau n'a-t-il pas fait remonter la thèse soutenue par cette note dans le corps du chapitre, et a-t-il ainsi minoré cette idée essentielle que la liberté est ce qui importe au citoyen et qu'elle détermine, en fin de compte, la prospérité du corps politique? Pourquoi, pour le dire autrement, se livre-t-il ainsi à une sorte de double jeu? Cette question est décisive pour notre compréhension de sa pensée politique.

\section{La force des choses et celle de la législation : la politique entre réel et possible.}

Pour donner toute sa consistance au texte que nous venons de lire, nous avons commencé par donner leur sens plein aux concepts de sujet et de citoyen: ils ne représentent pas des points de vue subjectifs entre lesquels on pourrait choisir, mais les deux positions dans lesquelles tout membre du corps politique se trouve effectivement. Ce constat a pour conséquence inévitable que les préférences du sujet, et les principes axiologiques qui les fondent, ne sont pas moins mais autrement fondés que ceux du citoyen. Ils sont l'expression du rapport spécifique au corps politique du membre de l'État, tandis que les valeurs du citoyen expriment sa position de membre du Souverain. Il faut revenir de ce point de vue sur le rapport que le sujet entretient avec la protection de ses biens ou, pour le dire d'un mot avec la propriété. Nous avons rappelé sa place dans la généalogie de la société civile établie par le second Discours. À deux reprises, Rousseau y souligne le rôle joué par la crédulité du peuple et la prise qu'elle donne aux discours fallacieux qui surprennent sa bonne foi. Une première fois, celui qui proclame "ceci est à moi » ne parvient à ses fins qu'en trouvant « des gens assez simples pour le croire ${ }^{48}$. De nouveau, les riches dont le but premier est de garantir leurs propriétés ne peuvent convaincre les pauvres de se soumettre à un gouvernement commun que parce qu'ils trouvent en eux «des hommes grossiers, faciles à séduire ${ }^{49}$. Pourtant, jamais Rousseau n'envisage de revenir sur l'institution de la propriété, ni sur celle du gouvernement. Il n'hésite pas même à dire, dans le Discours sur l'économie politique, que "le droit de propriété est le plus sacré de tous les droits des citoyens » ${ }^{50}$ et, dans le Contrat social, que les principaux avantages que l'homme retire de la

48. DOI, op. cit., p. I64.

49. DOI, op. cit., p. I77.

50. Discours sur l'économie politique, édition et commentaire sous la dir. de B. Bernardi, Vrin 2002, p. 65. Voir le commentaire d'I. Bouvignies, "Droit de propriété et domaine public", p. $173-193$. 
société civile sont «la liberté civile et la propriété de tout ce qu'il possède $»^{51}$. Le contraste entre ces deux séries de propositions, cela a été suffisamment établi, tient à ce que "juger les faits par le droit» ne veut en aucune façon dire substituer le droit au fait. Rousseau s'est toujours défendu d'être un utopiste. Pour lui, l'histoire de l'homme ne peut être refaite, le changement qui l'a affecté est irréversible: dans la société civile, les relations réelles déterminent les relations personnelles. Le problème qu'il se pose est de «rendre légitime» ce changement ${ }^{52}$. Or les réponses qu'il lui apporte reviennent bien souvent à chercher «le remède dans le mal » ${ }^{53}$. Preuve en est ici que le souverain s'assure de la fidélité des sujets par le pouvoir qu'il exerce sur leurs possessions devenues propriétés sous l'autorité de la loi. Il faut prendre toute la mesure de ce réalisme anthropologique. Dans une société «bien ordonnée », en devenant citoyen on ne cesse pas d'être sujet. L'intérêt commun ne supprime pas l'intérêt privé et la volonté générale n'efface pas celles des particuliers mais, ce qui est bien différent, la première doit prendre le pas sur les secondes chaque fois qu'elles pourraient être en contradiction. Doit-on en conclure que la politique du citoyen et celle du sujet sont toutes deux également légitimes?

Deux réponses doivent être apportées à cette question. La première sera positive dans la mesure où nous ne pouvons pas échapper à cette réalité: l'ordre social doit répondre à deux exigences également nécessaires. Mais ce sont deux types de nécessité différents. L'une est enracinée dans l'histoire de la société civile qui s'est formée pour, dans un même mouvement, assurer à chacun la possession de ses biens et mettre un terme au "plus horrible état guerre » que la première appropriation de la terre a engendré. L'autre répond à la nécessité de rendre ce changement légitime, c'est-à-dire à établir «la liberté politique et civile» et le degré d'égalité que celles-ci exigent. La tâche du gouvernement est donc à la fois d'assurer «la tranquillité publique", "la paix", et de maintenir "la liberté politique et civile». Ces "qualités morales" pouvant entrer en contradiction, il sera toujours nécessaire d'établir une pondération entre leurs "quantités» respectives, et l'évaluation de l'action du gouvernement sera toujours différente selon que l'on se placera du point de vue du sujet ou de celui du citoyen.

Mais est-il bien rigoureux de parler d'une politique du sujet comme on peut parler d'une politique du citoyen? Sans doute pas. Loin qu'il s'agisse d'une simple question de terminologie, nous avons affaire à ce qui est peutêtre le cœur du concept de politique pour Rousseau. Le point de vue du sujet se caractérise comme celui de la personne privée et du membre de l'État. Celui du citoyen, membre du souverain, est celui de la législation dont l'objet est le bien commun. L'un défend une situation acquise, l'autre décide

51. CS, I, VIII.

52. CS, I, I.

53. J. Starobinski, Le remède dans le mal, Gallimard, I989. 
des règles d'un ordre légitime. Évoquant l'exigence d'égalité sociale dont le principe de la liberté politique est porteur, Rousseau fait cette remarque décisive: "Cette égalité, disent-ils, est une chimère de spéculation qui ne peut exister dans la pratique: Mais si l'abus est inévitable, s’ensuit-il qu>il ne faille pas au moins le régler? Crest précisément parce que la force des choses tend toujours à détruire légalité, que la force de la législation doit toujours tendre à la maintenir ${ }^{54}$. Cette observation peut se généraliser: le point de vue du sujet, la tranquillité publique, est celui de la force des choses, le point de vue du citoyen, la liberté politique et civile est, autant qu'il est possible, de rendre cet ordre légitime. Celui-là seul est à proprement parler politique. Au demeurant, demander à la politique de faire contrepoids à la force des choses, loin de signifier un déni de cette dernière, exige d'en reconnaître la source et les ressorts pour en borner les effets. Ce qui est vrai de la tendance à «détruire l'égalité » portée en elle par la société civile l'est aussi de la tendance à "usurper la souveraineté » inhérente à toute institution politique. La seconde partie du livre III porte sur ce point: après avoir montré en tout gouvernement une "pente à dégénérer", Rousseau établit "comment se maintient l'autorité Souveraine»: par la seule activité du peuple assemblés5. Très logiquement, il reprend à ce propos ce qu'il disait au livre II au sujet de l'égalité: "Le peuple assemblé, dira-t-on! Quelle chimère! C'est une chimère aujourd'hui, mais ce n'en était pas une il y a deux mille ans: Les hommes ont-ils changé de nature? Les bornes du possible dans les choses morales sont moins étroites que nous ne pensons: Ce sont nos faiblesses, nos vices, nos préjugés qui les rétrécissent. Les âmes basses ne croient point aux grands hommes: de vils esclaves sourient d'un air moqueur à ce mot de liberté ».

Qu'il s'agisse de l'égalité ou de la liberté, la politique du citoyen consiste à ne pas s'incliner devant la force des choses mais à repousser, autant qu'il se peut, les bornes du possible ou, ce qui est une autre manière de le dire, à faire prévaloir les relations personnelles sur les relations réelles.

La rédaction de ce texte, entreprise après les attentats terroristes dont Paris a fait l'objet en janvier 2015, a été achevée dans les jours qui ont suivi ceux de novembre. Ces circonstances nous ont rappelé qu'il était nécessaire de tenir compte et de la priorité du sujet, la tranquillité publique, et de celle du citoyen, la liberté civile et politique, mais Rousseau nous incite à mesurer aussi, et peut-être surtout, que l'inversion de ces priorités signifierait l'abandon de la politique du citoyen.

Bruno Bernardi, le 30 novembre 2015

54. CS II, XI.

55. Voir respectivement CS III, X et XII. 